\title{
AIAA 2002-1368
}

Vibration of Prestressed Membrane Structures in Air

S. Kukathasan and S. Pellegrino

Department of Engineering, University of Cambridge,

Cambridge CB2 1PZ, UK

\section{3rd AIAA/ASME/ASCE/AHS/ASC Structures, Structural Dynamics, and Materials Conference 22-25 April 2002 Denver, $\mathbf{C O}$}

For permission to copy or republish, contact the American Institute of Aeronautics and Astronautics 1801 Alexander Bell Drive, Suite 500, Reston, VA 20191-4344 
AIAA-2002-1368

\title{
VIBRATION OF PRESTRESSED MEMBRANE STRUCTURES IN AIR
}

\author{
S. Kukathasan* and S. Pellegrino ${ }^{\dagger}$ \\ Department of Engineering, University of Cambridge \\ Trumpington Street, Cambridge, CB2 1PZ, U.K.
}

\begin{abstract}
$\underline{\text { Abstract }}$
Including air effects in the finite-element model of large deployable membrane structures can lead to considerable simplification in the ground testing of these structures, by removing the need to carry out extensive tests in vacuum chambers. This paper presents a finite-element modelling technique which simulates very accurately the air-membrane interaction. Results obtained with the ABAQUS package are compared with three test cases, which include a closed-form analytical solution and two sets of experiments.
\end{abstract}

\section{Introduction}

Thin membranes are increasingly being considered for large deployable space structures applications, due to the growing requirement for furlable reflecting surfaces for solar sails, space radars and reflector antennas. These structures are generally very flexible, and there is a need to have accurate models to predict their vibration behavior, which impacts their geometric accuracy and hence their performance, and may also lead to dynamic coupling with the attitude control system of the spacecraft.

It is well-known that large lightweight membrane structures vibrating in air have lower natural frequencies and higher damping than in vacuum, hence in general ground tests on these structures need to be done in a vacuum chamber. However, this is expensive and far from straightforward. For example, recent tests of a large membrane sunshield for the Next Generation Space Telescope ${ }^{1}$ posed considerable challenges, due to: (i) size limitations of existing vacuum chambers, (ii) difficulty of measuring whole model response through the glass portholes of the chamber, and (iii) difficulty of operating dynamic shakers, etc. under vacuum. Additional difficulties have been identified. ${ }^{2}$ Therefore, it is of interest to develop procedures whereby at least some experiments are con- ducted in air and validated against numerical models. Of course, the presence of air around the membrane needs to be properly included in these models. Once validated in this way, the numerical models could then be used to predict the vibration behavior in vacuum.

The vibration of a large membrane structure surrounded by an unbounded volume of air is a coupled fluid-structure interaction problem. Because the air displacements are small, the air can be treated as an acoustic medium which interacts with the vibrating surface through the acoustic pressure that it applies on it. Also, since we are only interested in finding the effects on the structure of the presence of the fluid, effects such as acoustic scattering or radiation in the fluid need not be considered.

The aim of this paper is to present a technique for modelling prestressed membrane structures vibrating in air by means of a standard finite element package. The accuracy of the model will be checked against three separate test cases, one based on a closed-form analytical solution and two based on experiments.

The layout of this paper is as follows. First, a brief review of relevant literature is presented. Second, a closed-form analytical solution is derived to estimate the natural frequencies of an infinite flat membrane in air. Third, experiments carried out on triangular, prestressed membranes to measure their resonant frequencies and corresponding mode shapes in air are presented. Fourth, a finite element modelling technique is presented and validated against the analytical solution and experimental results. A discussion concludes the paper.

\section{Background}

Most previous work on the vibration of elastic structures surrounded by a fluid has been focussed on plates; only recently has work specific to membranes been published. ${ }^{1-4}$ However, because the same general

\footnotetext{
* Research Student.

${ }^{\dagger}$ Professor of Structural Engineering, Associate Fellow AIAA. pellegrino@eng.cam.ac.uk Copyright (c) 2002 by S. Pellegrino. Published by the American Institute of Aeronautics and Astronautics, Inc. with permission.
} 
approach can be applied to both types of structures, it is appropriate to begin by reviewing some key results for plates. ${ }^{5}$

The vibration response of an infinite plate in an unbounded fluid medium changes at the so-called coincidence frequency. ${ }^{6}$ At this transition the fluid loading effect on the vibrating plate changes from providing a non-structural mass to providing radiation damping. When the plate vibrates below the coincidence frequency, it accelerates and compresses a small layer of fluid, ${ }^{5-8}$ whose thickness decreases as the frequency increases. An analytical solution can be obtained ${ }^{6}$ by assuming that the natural modes of the fluid loaded plate are identical to the modes in vacuum. Plates of finite size are slightly damped by the fluid even below the coincidence frequency, as well as showing an added mass effect. ${ }^{9}$ This is because edge effects cause acoustic radiation. ${ }^{8}$

Fluid-structure interaction problems are normally solved by combining a finite element (FE) model of the structure with a model of the fluid, computed using either FE or boundary element (BE) methods. In the case of a structure fully submerged in an infinite fluid medium, a FE representation of the fluid requires a very large mesh. Boundary element methods tend to be more attractive, as a $3-\mathrm{D}$ infinite medium can be modelled with 2-D surface elements. ${ }^{10-12}$ Reference $^{10}$ gives a thorough explanation of how to find the natural frequency of any structure submerged in an infinite air medium using a combined $\mathrm{FE} / \mathrm{BE}$ model. It also points out that achieving convergence to the natural frequency can prove troublesome; several iterations are often required. Two major disadvantages of BE methods ${ }^{13}$ are that they remain computationally expensive because of the non-bandedness of the stiffness matrix, in spite of the reduction in dimensionality, and also because the formulation becomes singular at the coincidence frequency. On the other hand, FE representations of an infinite fluid medium using a finite domain with carefully chosen boundaries have been shown to be possible, without affecting the acoustic field. ${ }^{14-15}$ Any artificial exterior acoustic boundaries should be placed above a minimum distance from the vibrating surface. The distance and type of the exterior boundaries need to be selected such that they do not interfere with the origin of the acoustic waves. ${ }^{16}$

Membranes have negligible out-of-plane stiffness, unless they are prestressed. A study of the effects of prestress and air pressure on the vibration of a triangular membrane with edge cords ${ }^{3}$ determined the natural frequencies and the corresponding mode shapes at different air pressures, i.e. in vacuum, at $0.61 \mathrm{~atm}$ and at 1 atm. A physical model was excited by an electrodynamic shaker attached to a corner of the triangle, and the dynamic response was measured using a non-contact capacitance device. These experiments indicate significantly lower and more closely spaced natural frequencies at higher air pressures, and also some distortion of the mode shapes in vacuum. The experimental results presented in this reference are a very valuable benchmark against which to test new solutions. FE predictions of the in vacuo mode shapes were obtained. An approximate analytical method was also proposed, which simulates air effects by adding a non structural mass equal to the volume of air contained in an envelope defined by the radius of the largest circle inscribed in the triangle. This method was able to predict the accuracy of the fundamental natural mode to an accuracy of $10 \%$ but was much less accurate for higher modes. For example, the sixth natural frequency in air was underestimated by $27 \%$ and the corresponding mode shape was also poorly estimated.

The lack of agreement between the experimental results and the approximate method in Ref. 3 is mainly due to the assumption of the same air mass for each mode. In reality, the air mass participating in the membrane vibration decreases with the increase of mode number ${ }^{6}$. This points to the need for a proper criterion for estimating the added mass. Several attempts at refining Ref. 3 were made at the early stages of the present study ${ }^{17}$ but, although some improvements were indeed achieved, it was concluded that simple geometric criteria cannot provide high accuracy.

\section{An Analytical Solution}

The scope for analytical solutions is very limited, hence we turn to the standard solution for an infinite flat plate surrounded by a fluid ${ }^{8}$ which can be adapted to infinite membranes, as shown next. It is assumed that the in vacuo modes are preserved in air. The coupling is achieved by assuming the same wavelength for the membrane and the air, and by matching the transverse response of the membrane to the air pressure at the interface.

\section{Membrane Vibration in Vacuum}

Consider a thin, flat membrane in an $x, y$ coordinate system. It is subject to biaxial tension $T$ per unit length, which will be assumed to remain constant when the membrane vibrates, and mass per unit area $m_{s}$.

Denoting by $w(x, y, t)$ the out-of-plane displacement of point $(x, y)$ at time $t$ and neglecting the bending stiffness of the membrane, the differential equation 
of motion has the form ${ }^{18}$

$$
T\left(\frac{\partial^{2} w}{\partial x^{2}}+\frac{\partial^{2} w}{\partial y^{2}}\right)=m_{s} \frac{\partial^{2} w}{\partial t^{2}}
$$

If the membrane is either infinitely long in both $x$ and $y$, or of finite dimensions but of rectangular shape, the general steady-state solution of Equation (1) is of the form

$$
w=\widetilde{w} e^{i \omega t} e^{-i\left(k_{s x} x+k_{s y} y\right)}
$$

where $\widetilde{w}$ is the displacement amplitude, $k_{s x}$ and $k_{s y}$ are the elastic wave number components along the $x$ and $y$ directions —of dimension $\left(\right.$ length $\left.^{-1}\right)$ - and $\omega$ is the particular frequency of interest. Note that the subscript $s$ stands for structure.

These wave number components are related to the corresponding wave lengths, $\lambda_{s x}$ and $\lambda_{s y}$, of the vibration mode shape in the $x$ and $y$ directions by

$$
k_{s x}=\frac{2 \pi}{\lambda_{s x}}, \quad k_{s y}=\frac{2 \pi}{\lambda_{s y}}
$$

Substituting Equation (2) into (1), we obtain

$$
k_{s x}^{2}+k_{s y}^{2}=\frac{m_{s} \omega^{2}}{T}
$$

and defining the elastic wave number, $k_{s}$, of the membrane at frequency $\omega$

$$
k_{s}^{2}=k_{s x}^{2}+k_{s y}^{2}
$$

we obtain

$$
\omega=k_{s} \sqrt{\frac{T}{m_{s}}}
$$

Note that this corresponds to a one-dimensional wave in a particular direction $\xi$, defined by the vector sum of $\lambda_{s x}$ and $\lambda_{s y}$, with wave length $\lambda_{s}=2 \pi / k_{s}$.

\section{Pressure Waves in Air}

Steady-state pressure waves in one or more dimensions, in a medium of density $\rho$ and bulk modulus $B$ satisfy the Helmholtz equation. ${ }^{7}$ We are particularly interested in two-dimensional waves, hence

$$
\left(\nabla^{2}+k_{a}^{2}\right) p=0
$$

where $p=p(\xi, \eta)$ is the pressure at point $(\xi, \eta)$, varying with time at frequency $\omega$, and $k_{a}$ is the acoustic wave number

$$
k_{a}=\omega \sqrt{\frac{\rho}{B}}
$$

The general solution of Equation (7) is a harmonic function of horizontal distance, $\xi$, and vertical distance, $\eta$, with acoustic wave numbers $k_{a \xi}$ and $k_{a \eta}$ in the two directions, hence

$$
p=\widetilde{p} e^{i \omega t} e^{-i\left(k_{a \xi} \xi+k_{a \eta} \eta\right)}
$$

Substituting Equation (9) into (7) leads to a relationship between the acoustic wave numbers that is analogous to Equation (5)

$$
k_{a}^{2}=k_{a \xi}^{2}+k_{a \eta}^{2}
$$

\section{Coupled Vibration}

The response of a membrane with air on one side only can be analysed as follows. The two solutions presented above, which are valid for any frequency $\omega$, are coupled by imposing that the acoustic wave component along the $\xi$-direction matches the structural wave along the $\xi$-direction. Therefore, the corresponding wave numbers must also match, hence

$$
k_{s}=k_{a \xi}
$$

It follows that from now on the $\eta$-direction will be perpendicular to the membrane and the corresponding wave number is then obtained by substituting Equation (11) into Equation (10). Noting that for thin membranes in air the elastic wave number $k_{s}$ is higher than the acoustic wave number $k_{a}$ gives

$$
k_{a \eta}=-i \sqrt{{k_{s}{ }^{2}-k_{a}{ }^{2}}^{2}}
$$

In the presence of air, Equation (1) becomes

$$
T\left(\frac{\partial^{2} w}{\partial x^{2}}+\frac{\partial^{2} w}{\partial y^{2}}\right)-p=m_{s} \frac{\partial^{2} w}{\partial t^{2}}
$$

where it can be shown ${ }^{7}$ that the pressure $p$ on the membrane is coupled to the motion of the membrane through

$$
\frac{\partial p}{\partial \eta}=-\rho \frac{\partial^{2} w}{\partial t^{2}} \quad \text { at } \quad \eta=0
$$

Differentiation of Equation (9) with respect to $\eta$ yields, after substituting Equation (9)

$$
\frac{\partial p}{\partial \eta}=-i k_{a \eta} p
$$

Then, substitution into Equation (14) gives

$$
p=\frac{\rho}{i k_{a \eta}} \frac{\partial^{2} w}{\partial t^{2}}
$$


Substituting Equation (16) into Equation (13),

$$
T\left(\frac{\partial^{2} w}{\partial x^{2}}+\frac{\partial^{2} w}{\partial y^{2}}\right)-\frac{\rho}{i k_{a \eta}} \frac{\partial^{2} w}{\partial t^{2}}=m_{s} \frac{\partial^{2} w}{\partial t^{2}}
$$

Substituting Equation (12) for $k_{a \eta}$ and re-arranging

$$
T\left(\frac{\partial^{2} w}{\partial x^{2}}+\frac{\partial^{2} w}{\partial y^{2}}\right)=\left(m_{s}+\frac{\rho}{\sqrt{k_{s}^{2}-k_{a}^{2}}}\right) \frac{\partial^{2} w}{\partial t^{2}}
$$

Thus, comparing Equation (18) with Equation (1) it can be seen that the presence of air introduces an additional air mass $m_{a}$ into the equation of motion, where

$$
m_{a}=\frac{\rho}{\sqrt{k_{s}^{2}-k_{a}^{2}}}, \quad \text { if } \quad k_{s}>k_{a}
$$

This amounts to saying that a layer of air of thickness $1 / \sqrt{k_{s}^{2}-k_{a}^{2}}$ vibrates together with the membrane. If $k_{s}<k_{a}$ the added air mass is imaginary, which means that air adds to the damping of the system. It is noted that when the frequency of vibration increases, then $k_{s}$ and $k_{a}$ increase proportionally, thus the air mass $m_{a}$ decreases.

In conclusion, an infinite membrane of mass $m_{s}$ per unit area which is subject to biaxial tension $T$ has infinitely many sinusoidal vibration modes. For any chosen mode, of wave lengths $\lambda_{s x}$ and $\lambda_{s y}$, we can calculate the wave number from Equations $(5,3)$

$$
k_{s}=2 \pi \sqrt{\frac{1}{\lambda_{s x}^{2}}+\frac{1}{\lambda_{s y}^{2}}}
$$

and the corresponding natural frequency of vibration in vacuum from Equation (6)

$$
\omega_{\text {vacuum }}=k_{s} \sqrt{\frac{T}{m_{s}}}
$$

The ratio of the wave numbers can be written, from Equations $(6,8)$, as

$$
\frac{k_{s}}{k_{a}}=\sqrt{\frac{m_{s}}{T} \frac{B}{\rho}}
$$

It is noted that this ratio is not a function of the frequency of vibration. Therefore, the type of fluid loading effect on the membrane does not change with the frequency of vibration. Hence, the nature of fluid loading on the membrane depends only on its mass per unit area and the prestress.

If the membrane vibrates in air, its mode shapes do not change but the natural frequency of vibration becomes

$$
\omega_{\mathrm{air}}=k_{s} \sqrt{\frac{T}{m_{s}+m_{a}}}
$$

where the air mass is iteratively calculated from

$$
m_{a}=\frac{\rho}{\sqrt{k_{s}^{2}-\omega_{\text {air }}^{2} \rho / B}}
$$

If there is air on both sides of the membrane, the same analysis can be repeated to obtain an equation like (24), but with a factor of 2 at numerator.

\section{An Experiment}

The natural frequencies and mode shapes of a triangular membrane vibrating in air were measured. A photograph of the experimental model is shown in Figure 1 and its dimensions are defined in Figure 2.

A triangular steel framework, rigidly fixed to the ground, was used to provide a rigid support for the membrane structure. The membrane itself is made of $0.05 \mathrm{~mm}$ thick aluminized Mylar foil, and has the shape of a right angle triangle with curved edges with sag-to-depth ratio is 20. A sleeve is formed along each edge of the foil, with Kapton tape, and Kevlar cords with a diameter of $0.92 \mathrm{~mm}$ are located inside the sleeve. Further details on the materials used are given in Table 1.

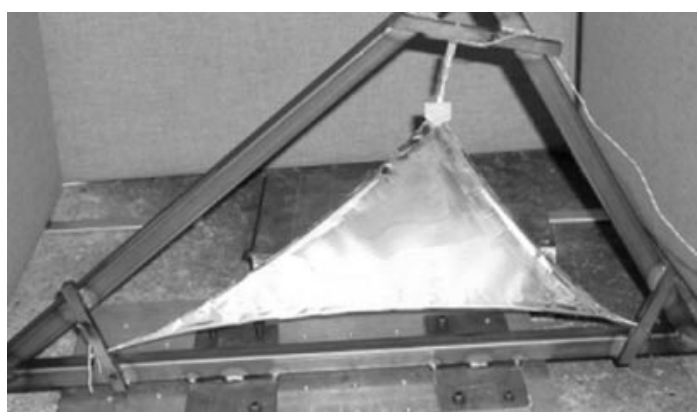

Figure 1: Experimental model.

Table 1: Material properties of experimental model.

\begin{tabular}{lccc}
\hline \hline & Membrane & Cord & Connector \\
\hline Material & Mylar & Kevlar & Al-alloy \\
Density $\left(\mathrm{kg} / \mathrm{m}^{3}\right)$ & 1070 & 1450 & 2700 \\
$\mathrm{E}\left(\mathrm{kN} / \mathrm{mm}^{2}\right)$ & 3.5 & 131 & 70 \\
$\nu$ & 0.35 & 0.30 & 0.33 \\
Mass $(\mathrm{g})$ & 5.1 & 1.3 & 12.2 \\
\hline \hline
\end{tabular}

The two cords that meet at each corner of the triangle are joined together with a steel loop (but a larger connector is used near the 90 deg corner) and glued with epoxy; then they are taken together to a bracket 
attached to the steel frame. Thus, in effect the membrane is connected to the frame by three cables, whose directions meet at a point, thus satisfying equilibrium. One of the three cables is connected to a strain-gauged turnbuckle; the shape of the triangle and the directions of the cables are such that the membrane is in uniform bi-axial prestress. All experiments reported in this paper were conducted at a uniform prestress of $20 \mathrm{~N} / \mathrm{m}$ in the membrane.

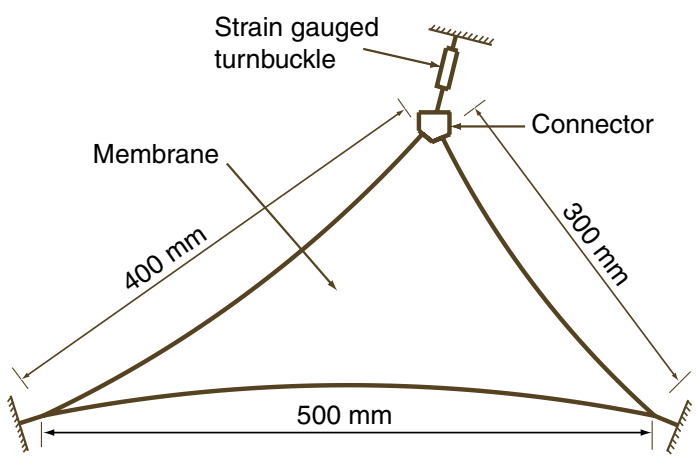

Figure 2: Schematic diagram of experimental model.

Modal identification tests were carried out, by hitting the surface of the connector with a PCB O86D80 impulse hammer fitted with a piezoelectric head, ${ }^{19}$ and by measuring the response of the membrane at 50 points with a Polytec PSV300 scanning laser vibrometer. Non-reflective powder had to be sprayed on the membrane surface, to avoid the laser beam from being deflected entirely away from the laser head. To reduce noise, each measurement was repeated five times and the results were averaged. After measuring all the target points, the Fast Fourier Transform (FFT) of the above signals was computed in order to obtain the frequency response function and the mode shapes of the model.

\section{Finite Element Modelling}

A key issue is how to set up an accurate and yet computationally manageable model of the air surrounding the membrane. Consider a square, flat membrane vibration with air on one side only, for simplicity. An air box of finite dimensions is defined above the membrane, as shown in Figure 3. The external boundary conditions around the box are chosen as follows.

Consider a pressure wave, $p$, propagating in the $\eta$ direction, i.e. vertically, away from the membrane. From Equations (9) and (12), for $k_{s}>k_{a} p$ has the expression

$$
p(\eta, t)=\widetilde{p} e^{i \omega t} e^{-\gamma \eta}
$$

where $\gamma=\sqrt{{k_{s}}^{2}-{k_{a}}^{2}}$.

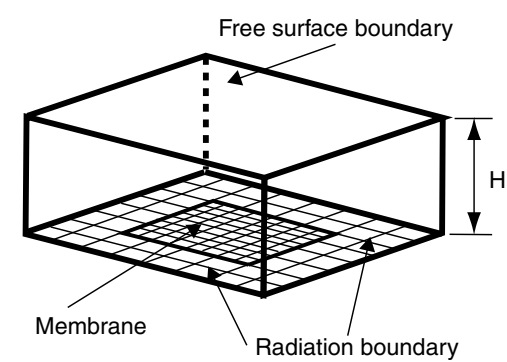

Figure 3: Finite element domain.

Since $p$ decays with distance from the membrane, a free surface boundary — with $p=0$ - can be placed at a certain height $H$ from the membrane surface. $H$ can be chosen such that the wave reflected by this free surface will have negligible effects when it reaches the membrane.

At the free surface the total pressure is zero. Therefore, considering both the incident wave $p_{i}$ and the reflected wave $p_{r}$

$$
p(H, t)=\widetilde{p}_{i} e^{i \omega t} e^{-\gamma H}+\widetilde{p_{r}} e^{i \omega t} e^{\gamma H}=0
$$

which gives,

$$
\widetilde{p_{r}}=-\widetilde{p_{i}} e^{-2 \gamma H}
$$

Now, the reflected wave that reaches the membrane surface is

$$
p_{r}(0, t)=-\widetilde{p}_{i} e^{-2 \gamma H} e^{i \omega t}
$$

The height $H$ of the air box will be selected such that $\widetilde{p_{r}} / \widetilde{p_{i}} \approx 0$, say $<1 / 400$. Hence

$$
e^{-2 \gamma H}<2.5 \times 10^{-3}
$$

Which, substituting

$$
\gamma \approx k_{s} \approx \frac{2 \pi}{\lambda_{s}}
$$

gives

$$
H \geq \frac{\lambda_{s}}{2}
$$

Here $\lambda_{s}$ is the wavelength of the fundamental mode of vibration of the membrane, or of any mode that is of particular interest.

The boundary conditions on the side faces of the air box are determined by considering a horizontal pressure wave. For $k_{s}>k_{a}$ Equations (9) and (11) give

$$
p(\xi, t)=\widetilde{p} e^{i \omega t} e^{-i k_{s} \xi}
$$


which shows that $p$ propagates without decaying. Therefore, the type of boundary condition that minimizes any interference is a radiation boundary. It has been found that boundaries of this type, placed on the sides of the air box, do not affect the frequency of the membrane if they are at a distance of no less than $\lambda_{s} / 2$ from the edges of the membrane.

\section{Choice of Elements}

The ABAQUS FE package ${ }^{20}$ was used throughout this study; here we give some details on the choice of elements. The membrane was modelled using 9node second-order quadrilateral membrane elements (M3D9) and the air domain with 3D solid acoustic elements, either 20-node second-order brick acoustic elements (AC3D20) or 15-node triangular prisms (AC3D15). These elements use acoustic pressure as a variable. The coupling between acoustic and membrane elements was set up by defining two surfaces along the interface, i.e. one lying on top of the membrane and the other at the bottom of the acoustic elements, and by tying together the nodes in the two surfaces (*TIE). An advantage of defining these surfaces is that the two element meshes do not need to have matching densities. The acoustic surface had to be defined as the "master" surface, because the model of the membrane was more refined. The horizontal surface around the membrane was modelled as a "rigid wall", which is the default boundary condition applied by ABAQUS.

\section{Analysis Details}

A complete analysis consists of two main steps. First, a static analysis in which the geometric stiffness of the membrane, without air, resulting from the prestress is calculated. Second, a dynamic analysis of the coupled air-membrane model which estimates the resonant frequencies and corresponding mode shapes.

Prestress of the membrane and any cords is defined as an initial stress (*INITIAL CONDITIONS, TYPE $=$ STRESS). It is best to begin the analysis with an equilibrium iteration; if the given prestress distribution is not in equilibrium, ABAQUS will find one, but it is important to check that the prestress level has not significantly decreased. Once the prestress is known in full, a geometrically non-linear static analysis is carried out (*STEP, NLGEOM), in which the geometric stiffness matrix is calculated. This geometric stiffness is automatically included in the following dynamic analysis.

The FE model of a coupled air-membrane problem has unsymmetric overall stiffness and mass matrices, although the eigenvalues are real. ${ }^{15}$ Therefore a complex eigensolver is needed, but this is not available in ABAQUS. ${ }^{20} \mathrm{~A}$ way around is to use a frequency response analysis instead (*STEP, *STEADY STATE DYNAMICS, DIRECT), by applying a unit, steadystate input load at a chosen node of the membrane and by varying the frequency over the range of interest. The responses of all membrane nodes to this input are recorded over the whole range of frequencies. The response amplitudes at a chosen node are plotted as a function of frequency and the peaks in this plot identify the resonant frequencies. The corresponding mode shapes are obtained from the real part of the response of each node. Because damping is small, out-of-phase effects are neglected.

Although computationally more expensive than mode-based steady-state dynamic analysis, this method is more accurate. A further advantage is that proportional material damping can be easily incorporated as complex mass and stiffness matrices are no problem.

\section{$\underline{\text { Three Test Cases }}$}

\section{Comparison with Analytical Solution}

Consider an infinite membrane with air on one side, vibrating in a mode with $\lambda_{s x}=\lambda_{s y}=0.4 \mathrm{~m}$, see Figure 4. Let the membrane be a $0.05 \mathrm{~mm}$ thick Mylar foil, mass per unit area of $0.0535 \mathrm{~kg} / \mathrm{m}^{2}$, with a uniform, biaxial prestress of $20 \mathrm{~N} / \mathrm{m}$. The properties of air are $\rho=1.2256 \mathrm{~kg} / \mathrm{m}^{3}$ and $B=1.42 \times 10^{5} \mathrm{~N} / \mathrm{m}^{2}$.

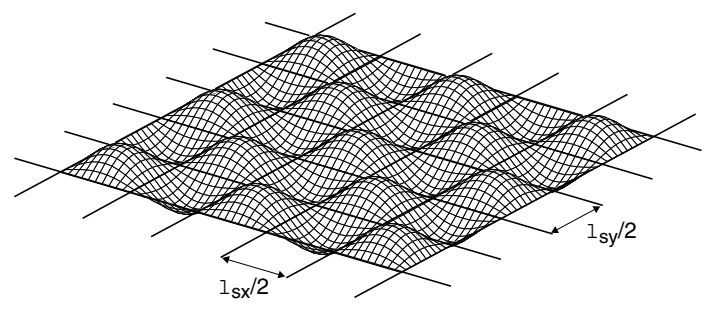

Figure 4: Chosen mode shape of infinite membrane.

From Equations (20) and (21) the in vacuo frequency is

$$
f_{\text {vacuum }}=68.36 \mathrm{~Hz}
$$

The ratio of wave numbers, from Equation (22) is

$$
\frac{k_{s}}{k_{a}}=17.8
$$

Therefore, air will act as a non-structural mass on the vibrating membrane. The frequency in air is estimated 
iteratively from Equations (23) and (24), as

$$
f_{\text {air }}=47.95 \mathrm{~Hz}
$$

Square membranes with side lengths of $0.2,0.6$, 1, 1.4, 1.8 and $2.2 \mathrm{~m}$, simply supported around all four edges and with uniform prestress, were defined in ABAQUS. Note that the dimensions of these membranes are all exact, odd multiples of the first; hence these structures will all admit a natural mode with the same wavelength of $\lambda_{s x}=\lambda_{s y}=0.4 \mathrm{~m}$. This mode has an analytical in vacuo frequency of $68.36 \mathrm{~Hz}$ and, for an infinite membrane, an analytical in air frequency of $47.95 \mathrm{~Hz}$.

In each case, a cubic air box was defined, as shown in Figure 3. The top and side faces of the box were placed at a distance of $0.15 \mathrm{~m}$ from the membrane; this is slightly higher than the value, $0.14 \mathrm{~m}$, obtained from Equation (28).

Element sizes were kept equal to $40 \mathrm{~mm}$ in all models, in order to focus on the effects of using a finite air box.

The ABAQUS analysis was divided into the following two steps:

- Step 1: All edge nodes of the membrane held fully constrained while an initial, non-linear equilibrium analysis was carried out by applying bi-axial uniform prestress of $20 \mathrm{~N} / \mathrm{m}$ to all membrane elements.

- Step 2: A linear frequency response analysis was carried out, initially between 40 and $50 \mathrm{~Hz}$ and then in a smaller range but with higher resolution. The excitation was applied at a non-central node of the membrane (to avoid symmetry effects).

For each membrane, a frequency response function was plotted and the mode shape corresponding to the observed peak was also plotted, to check that the correct mode had been identified. The natural frequencies obtained by this method for all six membranes have been plotted in Figure 5. The figure shows that the ABAQUS frequencies converge to the analytical frequency as the sidelength of the membrane is increased.

\section{Comparison with Experimental Results}

The experimental frequency response function in the range $0-100 \mathrm{~Hz}$ of the membrane structure described in the Section "An Experiment", obtained by averag- ing the response measured at 50 points, is plotted in Figure 6.

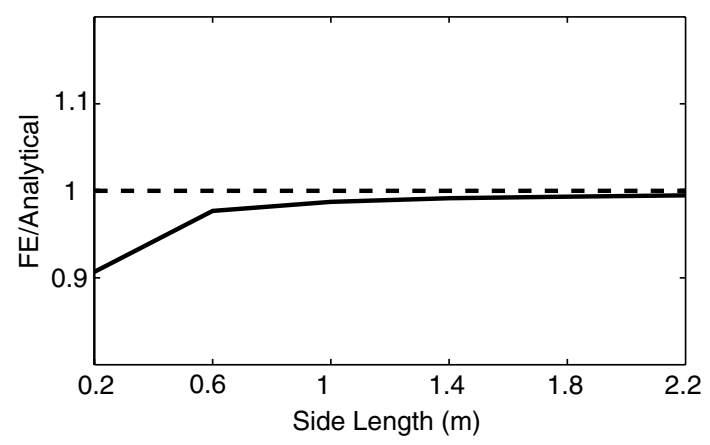

Figure 5: Ratio between FE and analytical frequencies for square membranes of different side lengths.

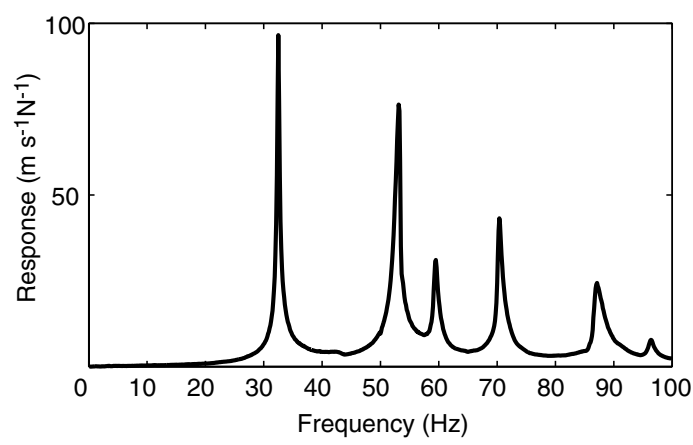

Figure 6: Experimental frequency response function of structure shown in Figure 1.

Table 2 lists the six resonant frequencies that were identified, together with the damping of each mode, expressed as a percentage of critical damping, obtained using the half-power method. ${ }^{21}$ Note that the first five modes are well separated, whereas the sixth is not. Also note that the damping values, ranging from 0.46 to $1.16 \%$, suggest that radiation damping is small.

Table 2: Experimental frequencies.

\begin{tabular}{ccc}
\hline \hline Mode & Frequency $(\mathrm{Hz})$ & Damping $(\%)$ \\
\hline 1 & 32.5 & 0.62 \\
2 & 52.5 & 1.16 \\
3 & 59.5 & 0.60 \\
4 & 70.2 & 0.46 \\
5 & 86.6 & 0.76 \\
6 & 96.2 & 0.49 \\
\hline \hline
\end{tabular}

Contour plots of the mode shapes obtained for each resonant frequency are shown in Figure 7. These mode shapes were estimated by taking the real part of the complex responses of each target point. 


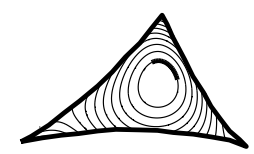

Mode 1 (32.5 Hz)

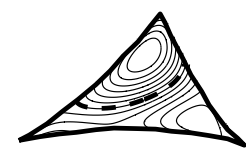

Mode 4 (70. $2 \mathrm{~Hz})$

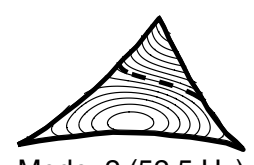

Mode $2(52.5 \mathrm{~Hz})$

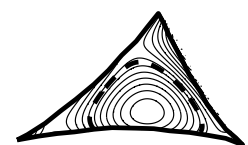

Mode $5(86.6 \mathrm{~Hz})$
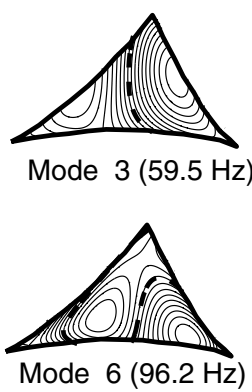

Figure 7: Experimental mode shapes (nodal lines shown broken).

An ABAQUS model of this structure was set up. In addition to the main elements, described previously, the cords were modelled with truss elements (T3D3). The connector was modelled as a rigid lamina (R3D3) of given mass and inertia, after it had been found that the use of acoustic elements around a small threedimensional body gives rise to numerical singularities. The air box was defined as a $0.6 \mathrm{~m}$ high hexagonal prism with the membrane in the middle; the minimum distance between the membrane and the boundaries, $0.3 \mathrm{~m}$, had been estimated from the fundamental frequency of the membrane in vacuum. The analysis was carried out with a mass proportional damping constant of $2.766 \mathrm{~s}^{-1}$ and also without damping.

This time the ABAQUS analysis was carried out in three steps:

- Step 1: All cable element nodes held fully constrained while a bi-axial, uniform prestress of 20 $\mathrm{N} / \mathrm{m}$ was applied to the membrane and the corresponding prestress was applied to the cables. This step sets up the correct prestress distribution but, to avoid numerical convergence problems, without allowing any stress transfer between the cable and the membrane.

- Step 2: A non-linear equilibrium static analysis was carried out after releasing all constraints on the cable nodes, apart from the nodes constrained to the edge structure. This step ensures that the cable nodes are in equilibrium.

- Step 3: A linear frequency response analysis was performed under a unit load applied at the connector, varying between 0 and $100 \mathrm{~Hz}$. The displacement and velocity responses of all nodes were computed.

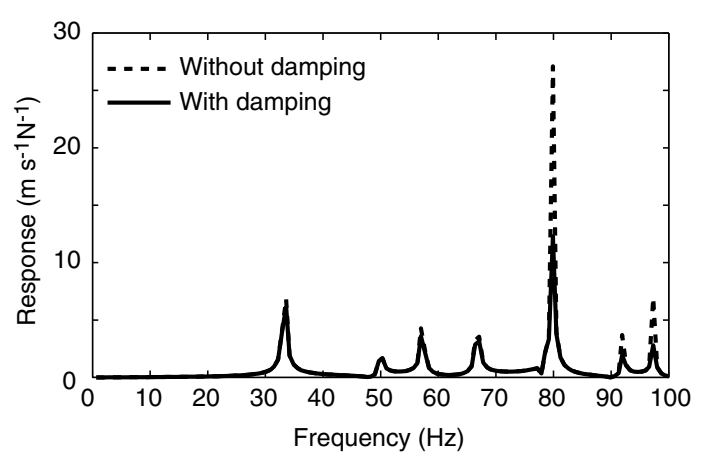

Figure 8: FE velocity response function.

Figure 8 shows the frequency response function for one particular node, near the right-hand-side corner of the triangle. Seven resonant frequencies are identified and the corresponding modes are plotted in Figure 9.
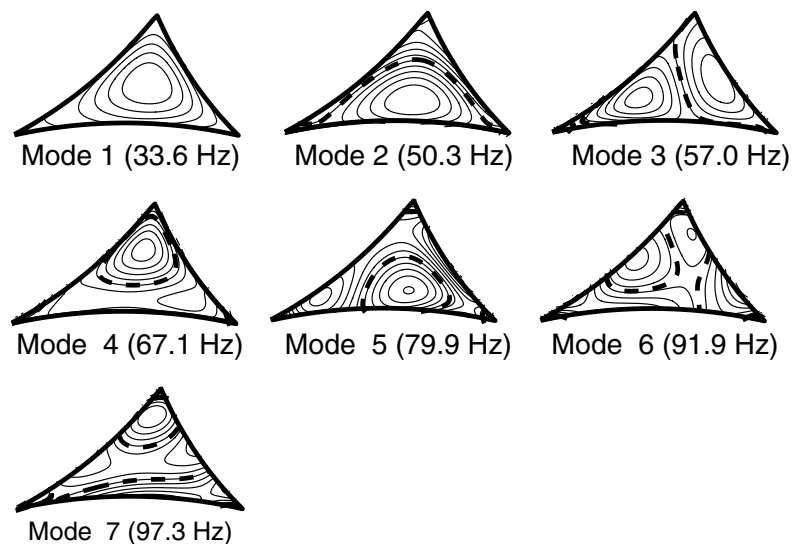

Figure 9: FE mode shapes (nodal lines shown broken).

Comparing the mode shapes in Figures 7 and 9, it is noted that the first five are in good agreement, but the sixth experimental mode corresponds to neither mode 6 nor made 7 from ABAQUS. Considering that the experimental frequency response function shows signs that other modes might exist between 90 and $100 \mathrm{~Hz}$, it seems likely that modal overlap occurs after the fifth mode. Therefore, only the first five modes are compared in Table 3.

Table 3: Comparison of experimental and FE results.

\begin{tabular}{ccccc}
\hline \hline \multirow{2}{*}{ Mode } & \multicolumn{2}{c}{ Natural Frequency $(\mathrm{Hz})$} & \multirow{2}{*}{ Error $(\%)$} \\
\cline { 2 - 4 } & FE vacuum & Experiment & FE air & \\
\hline 1 & 44.9 & 32.5 & 33.6 & 3.4 \\
2 & 60.1 & 52.5 & 50.3 & -4.2 \\
3 & 73.4 & 59.5 & 57.0 & -4.2 \\
4 & 84.4 & 70.2 & 67.1 & -4.4 \\
5 & 90.8 & 86.6 & 79.9 & -7.7 \\
\hline \hline
\end{tabular}

The first four predicted and measured frequencies

The velocity response function is plotted in Figure 8. 
differ by around $4 \%$ and the fifth by $7.7 \%$. Note that the difference between frequencies in air and in vacuum is highest $-12.4 \mathrm{~Hz}$, corresponding to a decrease of $26 \%$ - for the fundamental mode and decreases with the mode number.

\section{Comparison with Sewall's Experiment}

The third test case was based on one of the tests reported in Ref. 3. A schematic diagram of the model structure is shown in Figure 10, and the experimental mode shapes are shown in Figure 11.

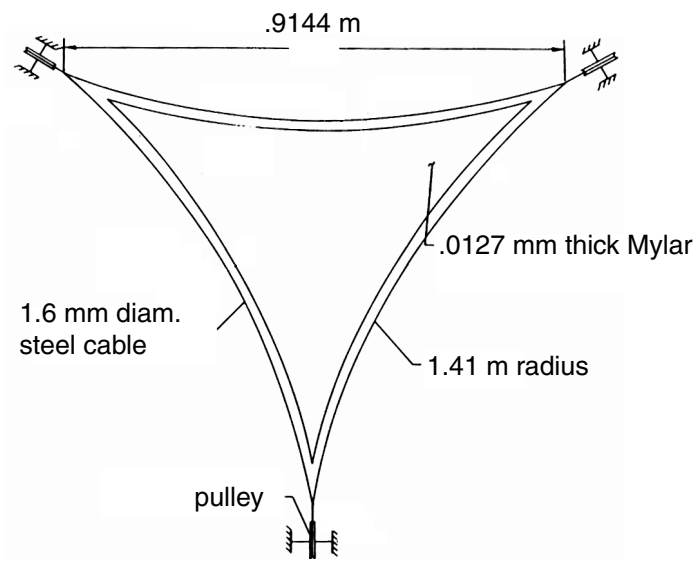

Figure 10: Membrane tested in Ref. 3.
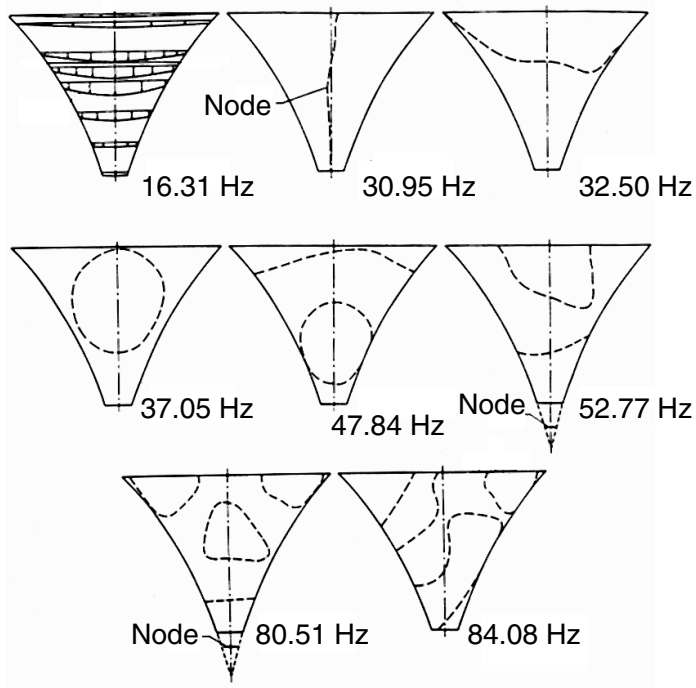

Figure 11: Experimental mode shapes at $93.4 \mathrm{~N}$ apex tension in air at 0.61 atm (from Ref. 3).

This structure was analysed with ABAQUS. The membrane and cables were modelled as described previously; the effect of the shaker was modelled as a point mass. A hexagonal air box, placed at $0.8 \mathrm{~m}$ from the membrane, was defined as discussed in the previous section.

The ABAQUS analysis had three steps, as for the previous test case, and the measured material damping ${ }^{3}$ was included as a mass proportional damping constant of $1.704 \mathrm{~s}^{-1}$. The frequency response function showed 12 modes between 0 and $100 \mathrm{~Hz}$. The nodal lines of the corresponding 12 mode shapes are plotted in Figure 12.

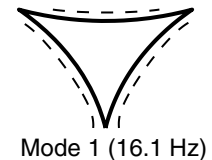

Mode $1(16.1 \mathrm{~Hz})$

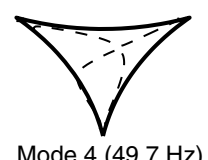

Mode $4(49.7 \mathrm{~Hz})$

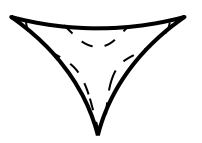

Mode $7(64.4 \mathrm{~Hz})$

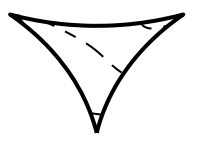

Mode $10(81.9 \mathrm{~Hz})$

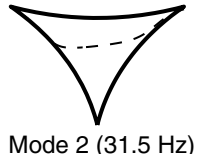

Mode $2(31.5 \mathrm{~Hz})$

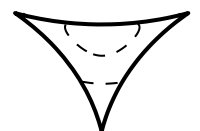

Mode $5(51.7 \mathrm{~Hz})$

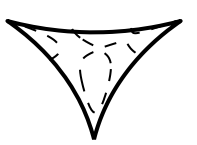

Mode $8(73.8 \mathrm{~Hz})$

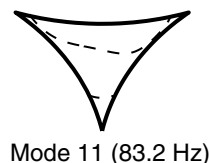

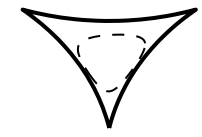

Mode $3(36.9 \mathrm{~Hz})$

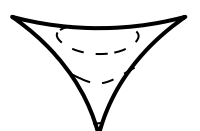

Mode $6(61.1 \mathrm{~Hz})$

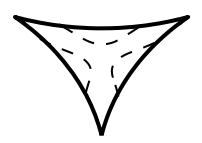

Mode $9(79.2 \mathrm{~Hz})$

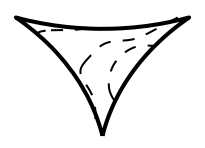

Mode $12(92.6 \mathrm{~Hz})$
Figure 12: FE mode shapes.

Comparing Figure 12 with Figure 11, the second experimental mode shape is missing. The reason is that this mode is anti-symmetric, and hence it is not excited by symmetric input applied to the ABAQUS model. The 2 nd, $3 \mathrm{rd}, 4$ th and 5 th modes from the finite element simulation are in fair agreement with the 3rd, 4th, 5th and 6th modes from the experiment, respectively. The differences in frequency are very small, from 0.5 to $4 \%$, see Table 4 .

Table 4: Comparison of experimental and FE results.

\begin{tabular}{cccc}
\hline \hline \multirow{2}{*}{ Mode } & \multicolumn{2}{c}{ Natural Frequency $(\mathrm{Hz})$} & \multirow{2}{*}{ Error $(\%)$} \\
\cline { 2 - 3 } & Experiment & FE air & \\
\hline 1 & 16.3 & 16.1 & 1.2 \\
2 & 30.9 & - & - \\
3 & 32.5 & 31.5 & 3.1 \\
4 & 37.1 & 36.9 & 0.5 \\
5 & 47.8 & 49.7 & -4.0 \\
6 & 52.8 & 51.7 & 2.1 \\
\hline \hline
\end{tabular}

\section{Discussion}

The analytical solution for the natural frequencies of infinite flat membranes vibrating in air has provided 
a good insight. When the structural wave number, $k_{s}$, is greater than the acoustic wave number, $k_{a}$, which is the typical case for thin membranes in air, it has been shown that a layer of air moves together with the vibrating membrane; this air thickness decreases with the increase in frequency of vibration. Therefore, the natural frequencies of the membrane in air are lower than its in vacuo frequencies because air acts as a non-structural mass, which is added to the mass of the membrane. In the three test cases presented in this paper the maximum difference was a decrease of $29 \%$ for one of the fundamental modes, but the higher modes show smaller differences.

Unlike thin plates vibrating in air, thin prestressed membranes do not show a coincidence frequency, because the ratio between structural and acoustic wave numbers is not a function of the frequency of vibration but only of the prestress and mass per unit area of the membrane.

In the experiment presented as our second test case we also had $k_{s}>>k_{a}$ and hence air acts mainly (but not only, due to edge effects) as an added mass. This is confirmed by the low damping that has been measured, between 0.4 and $1.2 \%$ of critical.

With this background, we have developed a FE modelling technique in which the membrane is enclosed in an air box of finite dimensions; these dimensions are related to the structural wavelength, $\lambda_{s}$. This modelling technique is reasonably economical of computer resources and has been shown to be very accurate by comparison with an analytical test case and two sets of experimental results. The first test has shown that the FE model converges to the analytical solution for an infinite membrane when the modelled size of the membrane is increased. The FE solution is within $2 \%$ of the analytical solution when a membrane size of 1.5 times the structural wavelength or greater is modelled. The second and third test cases, involving membrane structures that are representative of proposed applications, have shown that the frequencies obtained from the FE simulation are typically within $4 \%$ of the measured frequencies for our own experimental results, and within $2 \%$ of the published results. Considering that the physical model used for our experiments was about one-third the size of that used in Ref. 3, it seems reasonable to conclude that the higher level of error in the second test case is due to experimental error.

\section{$\underline{\text { Conclusion }}$}

The FE simulation technique presented in this paper predicts the natural frequencies and mode shapes of membrane structures vibrating in air with great ac- curacy. Hence, it is concluded that for model identification purposes it should be possible to reduce the amount of testing that needs to be carried out under vacuum.

\section{Acknowledgments}

We thank Dr W.R. Graham for helpful discussions of air-structure effects and Professor J. Woodhouse for help with the experiments.

\section{$\underline{\text { References }}$}

1 Lienard, S., Johnston, J.D., Ross B.P., and Smith, J., "Dynamic testing of a subscale sunshield for the Next Generation Space Telescope", Proc. 42nd AIAA/ASME/ASCE/AHS/ASC Structures, Structural Dynamics, and Materials Conference and Exhibit, Seattle, WA, 2001, AIAA-2001-1268.

2 Pappa, R.S., Lassiter, J.O., and Ross, B.P., "Structural dynamic experimental activities in ultralightweight and inflatable space structures", Proc. 42nd AIAA/ASME/ASCE/AHS/ASC Structures, Structural Dynamics, and Materials Conference and Exhibit, Seattle, WA, 2001, AIAA-2001-1263.

3 Sewall, J.L., Miserentino, R. and Pappa, R.S., "Vibration studies of a lightweight three-sided membrane suitable for space application", NASA TP 2095, 1983.

4 Johnston, J.D., and Lienard, S., "Modelling and analysis of structural dynamics for a onetenth scale model NGST sunshield" "Proc. 42nd AIAA/ASME/ASCE/AHS/ASC Structures, Structural Dynamics, and Materials Conference and Exhibit, Seattle, WA, 2001, AIAA-2001-1407.

5 Junger, M.C., "Acoustic Fluid-elastic structure interactions: basic concepts", Computers and Structures, Vol. 65, No. 3, 1997, pp. 287-293.

6 Junger, M.C., "Normal modes of submerged plates and shells", Symposium on Fluid-Solid Interaction, ASME, 1967, pp. 79-119.

7 Junger, M.C., and Feit, D., Sound, Structures, and Their Interaction, MIT Press, Massachusetts, 1986.

8 Fahy, F.J., Sound and Structural Vibration: Radiation, Transmission and Response, Academic Press Ltd., 1985.

${ }^{9}$ Davies, H.G., "Low frequency random excitation of water-loaded rectangular plates", Journal of Sound and Vibration Vol. 15, No. 1, 1971, pp. 107-126.

10 Sygulski, R. "Dynamic analysis of open membrane structures interacting with air", International Journal for Numerical Methods in Engineering, Vol. 37, 1994, pp. 1807-1823.

11 Zienkiewicz, O.C., Kelly, D.W., and Bettess, P. "The coupling of the finite element method and boundary solution procedures", International Journal for 
Numerical Methods in Engineering, Vol. 11, 1977, pp. 355-375.

12 Everstine, G.C., and Henderson, F. M., "Coupled finite element/boundary element approach for fluidstructure interaction", Journal of Acoustical Society of America, Vol. 87, No. 5, 1989, pp. 1938-1947.

13 Pinsky, P.M. and Abbound, N. N., "Two mixed variational principles for exterior fluid-structure interaction problems", Computers and Structures, Vol. 33, No. 3, 1989, pp. 621-635.

14 Zienkiewicz, O.C., and Newton, R.E., "Coupled vibrations of a structure submerged in a compressible fluid", Proc. Sym. Finite Element Techniques, 1969, pp. 359-379.

15 Everstine, G.C., "Finite element formulations of structural acoustics problems", Computers and Structures, Vol. 65, No. 3, 1997, pp. 307-321.

16 Zienkiewicz, O.C. and Bettess, P., "Fluid-structure dynamic interaction and wave forces: an introduction to numerical treatment", International Journal for Numerical Methods in Engineering, Vol. 13, 1978, pp. $1-16$.

17 Kukathasan, S. Vibration of prestressed membrane structures, M.Phil. Dissertation, University of Cambridge, 2000.

18 Rayleigh, J.W.S. The Theory of Sound, Vol. 2, Dover, New York, 1945.

19 Ewins, D.J., Modal Testing: Theory, Practice and Application, Research Studies Press Ltd., England, 2000.

20 Hibbitt, Karlsson and Sorensen Inc., ABAQUS Standard User's Manuals, Version 6.2, Pawtucket, RI, USA, 2001.

21 Meirovitch, L., Elements of Vibration Analysis, McGraw-Hill Inc., 1986. 